\title{
Efeito do extrato de tribulus terrestris na histomorfologia cardíaca e fisiologia testicular de ratos
}

\author{
Effect of tribulus terrestris extract on cardiac histomorphology and testicular physiology of rats \\ Efecto del extracto de tribulus terrestris sobre la histomorfología cardíaca y la fisiología testicular
}

de ratas

Recebido: 23/07/2021 | Revisado: 27/07/2021 | Aceito: 03/08/2021 | Publicado: 08/08/2021

Leonardo Luca Luciano

ORCID: https://orcid.org/0000-0001-8359-3558

Universidade Federal de Alfenas, Brasil

E-mail: leonardo.luciano@sou.unifal-mg.edu.br

Evelise Aline Soares

ORCID: https://orcid.org/0000-0001-7838-687X

Universidade Federal de Alfenas, Brasil

E-mail: evelise.anatomia@gmail.com

Samuel José Fernandes de Lima

ORCID: https://orcid.org/0000-0001-9725-1284

Universidade José do Rosário Vellano, Brasil

E-mail: samuelfvet@hotmail.com

Rayzza Tomaz Griebeler

ORCID: https://orcid.org/0000-0002-6455-5596

Universidade José do Rosário Vellano, Brasil

E-mail: rayzzagriebeler@gmail.com

Raissa Forgerini

ORCID: https://orcid.org/0000-0002-8534-2557

Universidade José do Rosário Vellano, Brasil

E-mail: raissaforgerini.93@gmail.com

Michelli Pereira

ORCID: https://orcid.org/0000-0002-1203-8757

Universidade José do Rosário Vellano, Brasil E-mail: michellipr1981@gmail.com

Ester Caixeta

ORCID: https://orcid.org/0000-0001-7780-8436 Universidade Federal de Alfenas, Brasil E-mail: ecaixeta@gmail.com

Vitória Lima Boaventura

ORCID: https://orcid.org/0000-0002-2412-2470

Universidade José do Rosário Vellano, Brasil E-mail: vitboaventura@hotmail.com

Diego Furtado da Cunha

ORCID: https://orcid.org/0000-0002-6043-4471

Universidade José do Rosário Vellano, Brasil

E-mail: diegomedxxv@gmail.com

Gérsika Bittencourt Santos Barros

ORCID: https://orcid.org/0000-0003-0849-2786

Universidade José do Rosário Vellano, Brasil

E-mail: gersika.santos@unifenas.br

José Antonio Dias Garcia

ORCID: https://orcid.org/0000-0002-4024-3045

Universidade José do Rosário Vellano, Brasil Instituto Federal de Educação Ciência e Tecnologia do Sul de Minas Gerais, Brasil

E-mail: jadiasgarcia@gmail.com

\section{Resumo}

O Tribulus terrestris (TT) é uma planta originária da Índia, conhecida como Abrolo ou "Videira-de-punctura". São muitas as incertezas relacionadas aos efeitos do TT na potência física de atleta, nos níveis de testosterona e como afrodisíaco. Há poucos relatos do efeito do TT no sistema cardiovascular, contudo citam que pode existir uma correlação negativa entre consumo do TT e atividade da enzima conversora de angiotensina. Neste estudo avaliamos o efeito do TT no ganho de peso, na concentração sérica da testosterona e na morfometria cardíaca de ratos. Trinca ratos foram divididos aleatoriamente dividido em 2 grupos experimentais $(n=15)$ : Grupo CT, tratados com solução 
fisiológica 0,9\%; grupo TT, tratados com extrato de Tribulus terrestris. Após 60 dias de tratamento, os animais que receberam TT ganharam menos peso corporal e não apresentaram diferenças nos níveis séricos de testosterona e morfometria cardíaca quando comparados aos ratos do grupo CT. Nossos resultados sugerem que o TT preveniu ganho de peso sem alterar o equilíbrio energético da dieta, sem influenciar a secreção da testosterona e sem alterar a morfometria cardíaca. Portanto, o TT pode ter um futuro promissor em preservar o peso corporal, tornando a prevenção do ganho de peso mais fácil de alcançar do que perder peso e manter essa perda.

Palavras-chave: Testosterona; Coração; Ganho de peso; Testículo.

\begin{abstract}
Tribulus terrestris (TT) is a plant native to India, known as Abrolo or "Puncture Vine". There are many uncertainties related to the effects of TT on the athlete's physical power, testosterone levels and as an aphrodisiac. There are few reports on the effect of TT on the cardiovascular system, however they mention that there may be a negative correlation between TT and the activity of the angiotensin-converting enzyme. In this study we evaluated the effect of TT without weight gain on serum testosterone concentration and cardiac morphometry in rats. Three rats were randomly divided into 2 experimental groups $(n=15)$ : Group CT, treated with $0.9 \%$ saline solution; TT group, treated with Tribulus terrestris extract. After 60 days of treatment, animals that received TT gained less body weight and did not dissipate serum testosterone levels and cardiac morphometry when compared to rats in the CT group. Our results obtained that TT prevented weight gain without altering the energy balance of the diet, without altering testosterone secretion and without altering cardiac morphometry. Therefore, TT may have a promising future in preserving body weight, making preventing weight gain easier to achieve than losing weight and maintaining that loss.
\end{abstract}

Keywords: Testosterone; Heart; Weight gain; Testis.

\title{
Resumen
}

Tribulus terrestris (TT) es una planta originaria de la India, conocida como Abrolo o "Puncture Vine". Existen muchas incertidumbres relacionadas con los efectos del TT en la potencia física del atleta, los niveles de testosterona y como afrodisíaco. Hay pocos informes sobre el efecto de la TT en el sistema cardiovascular; sin embargo, mencionan que puede haber una correlación negativa entre la TT y la actividad de la enzima convertidora de angiotensina. En este estudio evaluamos el efecto de TT sin aumento de peso sobre la concentración sérica de testosterona y la morfometría cardíaca en ratas. Tres ratas se dividieron aleatoriamente en 2 grupos experimentales $(n=15)$ : Grupo CT, tratado con solución salina al 0,9\%; Grupo TT, tratado con extracto de Tribulus terrestris. Después de 60 días de tratamiento, los animales que recibieron TT ganaron menos peso corporal y no disiparon los niveles séricos de testosterona ni la morfometría cardíaca en comparación con las ratas del grupo CT. Nuestros resultados obtuvieron que el TT evitó el aumento de peso sin alterar el balance energético de la dieta, sin alterar la secreción de testosterona y sin alterar la morfometría cardíaca. Por lo tanto, el TT puede tener un futuro prometedor en la preservación del peso corporal, lo que hace que sea más fácil prevenir el aumento de peso que perder y mantener esa pérdida.

Palabras clave: Testosterona; Corazón; Aumento de peso; Testículos.

\section{Introdução}

O Tribulus terrestris (TT) é uma erva rasteira perene, que é amplamente distribuída no Mediterrâneo e em regiões de clima tropical e desértico (Do et al., 2013), ela pertencente à família Zygophyllaceae e possui outros nomes populares como “videira da punctura", “abrojo", "viagra natural” entre outros (Hussain et al., 2009; Shahid et al., 2016). Essa erva, é utilizada na medicina popular como tônico, afrodisíaco, analgésico, anti-hipertensivo, diurético, antiurolítica, imunomoduladora, antidiabética, hipolipidêmica, cardiotônica, hepatoprotetora, anti-inflamatória, no tratamento de infecções do trato urinário, tratamento da impotência, disfunção sexual de mulheres na menopausa, como estimulante para ajudar a aumentar o desempenho sexual e a performance física e resistência (Hussain et al., 2009; Lemos Júnior et al., 2011; Chhatre el al., 2014; Souza et al., 2020). Estudos recentes demonstraram que o TT protege e restaura células pigmentares da retina humana contra o estresse oxidativo e possui efeitos benéficos na prevenção e tratamento de doenças cardiovasculares, função sexual masculina e diabetes (GamalEl Din et al., 2018; Reshma et al., 2019; Zhang et al., 2019; Yuan et al., 2020).

O uso da TT e os seus efeitos no organismo ainda é um assunto muito debatido e controverso, principalmente no que diz respeito a um efeito androgênico e na força muscular e composição corporal (Lemos Júnior et al., 2011).

Estudos de Wang et al. (1990) demonstraram que o TT seria um dos medicamentos ideais para o tratamento de angina de peito, pois mostraram o TT tem ação na dilatação da artéria coronária, com consequente melhora da circulação coronária e efeitos positivos no ECG de pacientes que sofreram isquemia do miocárdio. Guo et al. (2007) demostraram que o TT não só 
melhorou o perfil lipídico mas também aliviou o remodelamento ventricular esquerdo e a função cardíaca na fase inicial após o insulto miocárdio e a remodelação ventricular após infarto do miocárdio em ratos hiperlipidêmicos. Nessa perspectiva, Reshma et al. (2019) observaram em modelos in vitro e in vivo que o TT possui potencial anti-apoptótico durante o insulto isquêmico por meio da via de sinalização ativada por mitógenos.

Ervas como a TT estão se tornando cada vez mais atraente e popular entre a população ocidental devido a atividades de marketing intenso (Pokrywka et al., 2014). No entanto, é importante salientar que possuem alguns efeitos colaterais evidenciados na literatura. Dentre eles, incluem danos na produção de testosterona, aumento das doenças cardiovasculares, depressão, hipercalcemia, ginecomastia, distúrbios de fertilidade e alteração na espermatogênese (Samaha et al., 2008).

Embora inúmeros efeitos da TT em animais (Arcasoy et al., 1998; Gauthaman et al., 2002; Gauthaman e Ganesan, 2008; e humanos (Brown et al., 2000; Antonio et al., 2000; Kohut et al., 2003; Samani et al. 2016; GamalEl Din et al. 2020) tenham sido avaliados e muitos compostos ativos a partir de extrato de TT tenham sido estabelecidos, o modo de sua ação e eficácia ainda são incertos e controversos.

Devido à escassez de trabalhos que correlacione os efeitos da TT sobre os níveis de testosterona e suas consequências na morfologia cardíaca, o presente estudo teve como objetivo avaliar o efeito do TT sobre o ganho de peso, o consumo de ração, a histomorfologia ventricular esquerda e fisiologia testicular em ratos wistar.

\section{Metodologia}

\subsection{Aspectos éticos}

Este estudo foi aprovado sob parecer de $\mathrm{n}^{\circ} 25 \mathrm{~A} / 2014$ e todas as etapas foram conduzidos de acordo com Comitê de Ética em Pesquisa da Universidade José do Rosário Vellano (UNIFENAS).

Para a realização deste estudo, utilizou-se uma metodologia de natureza quantitativa em modelo experimental (Pereira et al., 2018).

\subsection{Animais do estudo}

$\mathrm{O}$ experimento foi realizado com $30 \mathrm{em}$ ratos Wistar albinos (Rattus norvegicus albinus) machos, adultos, com peso médio de $\pm 300 \mathrm{~g}$, fornecidos pelo Biotério Central da Universidade José do Rosário Vellano- UNIFENAS, Alfenas-MG, mantidos no Laboratório de Experimentação Animal da UNIFENAS em estantes ventiladas (Alesco, Monte Mor, SP, Brasil), com temperatura controlada de $25 \pm 2^{\circ} \mathrm{C}$ e regime de luz com ciclo claro-escuro de $12 \mathrm{~h}$. Os animais foram aleatoriamente divididos em 2 grupos experimentais ( $\mathrm{n}=15)$, assim distribuídos:

Grupo controle (CT) - tratados com solução fisiológica $0,9 \%$, por via oral, em um volume de $0,5 \mathrm{ml}$.

Grupo Tribulus terrestres (TT) - tratados com extrato de Tribulus terrestris, por via oral, na dose de $50 \mathrm{mg} / \mathrm{kg}$ em um volume de $0,5 \mathrm{ml}$ (solução), por um período de 60 dias ( 8 semanas).

\subsection{Monitoramento do consumo líquido e sólido}

A todos animais foi permitido o acesso livre a água e a dieta sólida comercial balanceada (Nuvilab CR - Nuvital, Colombo - PR, Brasil) e a dieta líquida foi oferecida em frascos de $500 \mathrm{~mL}$ para consumo livre. A cada 48 horas, as dietas sólida e líquida foram alteradas e os valores residuais foram registrados; além disso, os animais foram pesados semanalmente.

\subsection{Protocolo de eutanásia e coleta material biológico}

Após 60 dias de tratamento os animais foram pesados e posteriormente anestesiados seguindo o protocolo anestésico com Cloridrato de Xilazina (Rompun® 2g/10mL) - 40mg ( $0,2 \mathrm{ml})$, intraperitoneal em associação com Cloridrato de Ketamina 
(Ketamin $\left.{ }^{\circledR} 50 \mathrm{mg} / \mathrm{mL}\right)-10 \mathrm{mg}(0,2 \mathrm{ml})$, intraperitoneal. Logo após a remoção, o coração foi dissecado e o ventrículo esquerdo (VE) isolado. Para determinar a existência de hipertrofia ventricular esquerda foi calculada a proporção do peso ventricular esquerdo (mg) pelo peso corporal (g).

A concentração de testosterona no soro foi medida por enzimoimunoensaio (ELISA) como descrito nas instruções do Kit (Diagnostics biochemInc). Os testículos foram extraídos cirurgicamente e medidos no sentido longitudinal e transversal, pesados em balança analítica e calculado a razão entre o peso testicular (mg) e o peso do animal (g). O sêmen foi coletado após dissecação das estruturas testiculares, evidenciando o epidídimo para a dissecação do mesmo, e pressionando o epidídimo (sentido cabeça-cauda) em direção ao ducto deferente, com o uso de uma pinça anatômica. Os espermatozoides coletados foram transferidos para $50 \mu \mathrm{L}$ de solução fisiológica a $0,9 \%$ em placa de Petri previamente aquecida a $36^{\circ} \mathrm{C}$. Desta diluição foram coletados 5 microlitros e transferidos para lâmina sob lamínula para análise instantânea de motilidade e vigor. A avaliação da motilidade e vigor foi realizada através de microscopia óptica em aumento de 200 vezes. A motilidade foi avaliada em uma escala de 0 a $100 \%$ e o vigor em uma escala de 1 a 5 . A concentração espermática foi verificada em microscópio óptico a partir de uma alíquota de $20 \mu l$ da diluição inicial que, posteriormente, foi transferida para o volume de 6 $\mathrm{mL}$ de água destilada para imobilização dos espermatozoides. Para a análise microscópica da concentração espermática foi utilizada a câmara de Neubauer. As áreas superiores e inferiores da câmara de Neubauer foram preenchidas com a amostra avaliada. O número de espermatozoides foi calculado pela média dos valores encontrados nos quatro quadrantes laterais de cada área, sendo o número de células encontradas, multiplicado por dez à sexta (número de células x106).

\subsection{Processamento histológico e análise morfométrica}

O VE foi fixado por vinte e quatro horas em formalina a $10 \%$ juntamente com a artéria aorta. Em seguida, os órgãos foram incluídos em parafina para cortes histológicos de quatro micrômetros de espessura, segundo Junqueira et al. (1979). Cortes histológicos dos ventrículos foram corados com picrosirius red para avaliar e quantificar o colágeno do tecido cardíaco. Outros cortes histológicos dos ventrículos foram corados com hematoxilina/eosina para análise morfométrica dos cardiomiócitos. Foi obtido quatro fotomicrografias (200x), a partir de um mesmo ponto pré-fixado dos cortes transversais do ventrículo de cada camundongo, utilizando-se a câmera digital acoplada ao programa Leica IM50 (versão 1.20). Foram medidos os diâmetros de 8 a 12 cardiomiócitos de cada corte histológicos, totalizando doze cortes histológicos em cada animal (Armstrong et al., 1998). Os cortes corados com picrosírius red foram analisados com luz polarizada. Cada fotomicrografia foi analisada pelo software LGMC-image versão 1.0, por meio do qual foi adquirido as porcentagens fracionais de colágeno referentes às áreas marcadas em vermelho (Shirani et al. 2000). Todas as análises histológicas foram executadas por um único examinador, utilizando o método duplo cego.

\subsection{Análise Estatística}

Para a análise estatística dos resultados foi utilizado o Programa GraphPad Prism 5, e foi aplicado a Análise de variância (ANOVA) a 5\% de significância, apropriado para comparação de grupos independentes por meio do teste T.

\section{Resultados}

No presente estudo observou-se que os animais que receberam TT ganharam menos peso corporal quando comparados aos CT, com o mesmo consumo de ração e água e sem apresentarem diferenças nos níveis séricos da testosterona (Tabela 1). Quanto aos parâmetros avaliados na análise morfométrica cardíaca, o TT não influenciou na proporção peso cardíaco $(\mathrm{mg})$ pelo peso do animal $(\mathrm{g})$; diâmetro dos cardiomiócitos $(\mu \mathrm{m})$ e porcentagem de depósito de colágeno ventricular (\%) quando comparado aos ratos do grupo CT (Tabela 1 e Figura 1). 
Tabela 1. Efeitos do TT no peso, consumo médio de água e ração, concentração sérica de testosterona e análise morfométrica ventricular esquerda em ratos Wistar. CT, grupo controle; TT, grupo Tribulus terrestres.

\begin{tabular}{|c|c|c|c|}
\hline Parâmetros & $\mathbf{C T}$ & TT & Valor $\mathbf{p}$ \\
\hline $\mathrm{n}$ & 15 & 15 & \\
\hline Peso inicial dos ratos $(\mathrm{g})$ & $247 \pm 11^{\mathrm{a}}$ & $232 \pm 4^{\mathrm{a}}$ & 0,2057 \\
\hline Peso final dos ratos $(\mathrm{g})$ & $401 \pm 14^{\mathrm{a}}$ & $358 \pm 11^{\mathrm{b}}$ & $0,0144^{*}$ \\
\hline Ganho de peso durante 60 dias $(\mathrm{g})$ & $181 \pm 21^{a}$ & $132 \pm 12^{\mathrm{b}}$ & $0,0436^{*}$ \\
\hline Consumo médio diário de ração $(\mathrm{g})$ & $32,4 \pm 0,5^{\mathrm{a}}$ & $31,7 \pm 0,4^{\mathrm{a}}$ & 0,3238 \\
\hline Consumo médio diário de água $(\mathrm{mL})$ & $24 \pm 0,4^{\mathrm{a}}$ & $24,7 \pm 0,5^{\mathrm{a}}$ & 0,2813 \\
\hline Proporção peso cardíaco (mg) / peso do animal (g) & $1,83 \pm 0,04^{\mathrm{a}}$ & $1,99 \pm 0,07^{\mathrm{a}}$ & 0,0719 \\
\hline \multicolumn{4}{|l|}{$\mathrm{PC} / \mathrm{PA}$} \\
\hline Diâmetro dos cardiomiócitos $(\mu \mathrm{m})$ & $22 \pm 0,5^{\mathrm{a}}$ & $22 \pm 0,6^{\mathrm{a}}$ & 0,7210 \\
\hline Depósito de colágeno ventricular (\%) & $5,7 \pm 0,4^{\mathrm{a}}$ & $5,4 \pm 0,3^{\mathrm{a}}$ & 0,4980 \\
\hline Concentração sérica de testosterona (ng/dL) & $412 \pm 65^{\mathrm{a}}$ & $340 \pm 33^{\mathrm{a}}$ & 0,3063 \\
\hline
\end{tabular}

Os valores foram expressos como média \pm Erro padrão da média.

Médias seguidas de mesmas letras minúsculas na linha não diferem entre si pelo teste $t$-Student a $5 \%$ de probabilidade.

Fonte: Autores.

Figura 1. Fotomicrografias do ventrículo esquerdo (VE) após 60 dias de experimento. Coloração com hematoxilina e eosina (HE) nos ventrículos esquerdos, evidenciando o diâmetro dos cardiomiócitos, corados com HE. Fotomicrografias mostrando a distribuição da coloração intersticial e perivascular com colágeno vermelho (marcado em vermelho pelo corante) no miocárdio do ventrículo esquerdo de camundongos corados com picrosirius. CT, grupo controle; TT, grupo Tribulus terrestres.
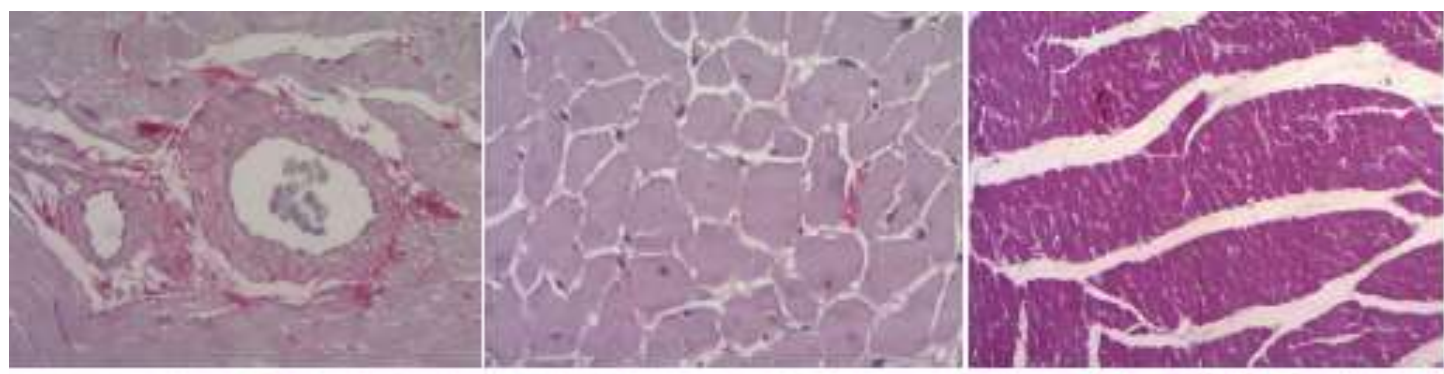

CT
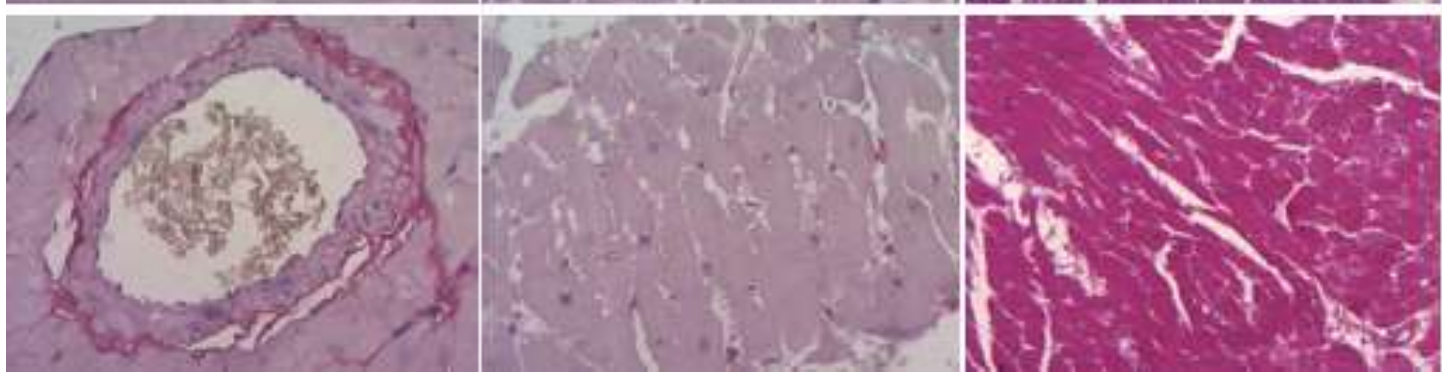

$\pi$

Fonte: Autores.

Em relação aos parâmetros do espermograma que avaliam a fertilidade dos camundongos foi observado aumento da motilidade e diminuição da concentração espermática. Não houve diferenças significativas no vigor (Tabela 2). Esses parâmetros podem ser correlacionados à análise da testosterona sérica evidenciada na Tabela 1. 
Tabela 2. Comparação da concentração espermática, motilidade e vigor dos espermatozoides de acordo com o espermograma de ratos Wistar. CT, grupo controle; TT, grupo Tribulus terrestres.

\begin{tabular}{cccc}
\hline Parâmetros & CT & TT & 15 \\
\hline $\mathrm{N}$ & $28,02 \pm 5,9^{\mathrm{a}}$ & 15 \\
\hline Concentração espermática $\left(x 10^{6} / \mathrm{ml}\right)$ & $54,40 \pm 5,04^{\mathrm{a}}$ & $21,6 \pm 3,73^{\mathrm{a}}$ & $58 \pm 5,87^{\mathrm{a}}$ \\
\hline Motilidade $(0-100 \%)$ & $2,47 \pm 0,19^{\mathrm{a}}$ & 0,3590 \\
\hline Vigor $(1-5)$ & $2,4 \pm 0,21^{\mathrm{a}}$ & \\
\hline
\end{tabular}

Os valores foram expressos como média \pm erro padrão da média. Médias seguidas de mesmas letras na linha não diferem entre si pelo teste $t$ Student a 5\% de probabilidade.

Fonte: Autores.

\section{Discussão}

O controle do consumo de ração e água durante o experimento foi fundamental, pois a desnutrição ou a desidratação mediante ao uso experimental do TT podem influenciar negativamente nos resultados da pesquisa. Os ratos dos grupos CT e TT, apresentaram consumos superiores a $25 \mathrm{~g}$ de ração/dia e acima de $15 \mathrm{~mL}$ de água/dia, valores preconizados como ideais pelos autores Gilberts e Wereldsma (2002) e Dahabra e Sawaqed (2007). Assim, os animais participantes do presente estudo não apresentaram desnutrição e desidratação, os animais do grupo CT e TT, estatisticamente apresentaram os mesmos consumos de água e ração, porém os animais do grupo TT apresentaram menor ganho de peso ao longo do experimento. Esses resultados corroboram com o estudo duplo-cego Rogerson et al. (2007) no qual o TT na dose de 450 mg/dia não produziu grandes ganhos de força ou massa muscular magra em relação ao placebo durante 5 semanas de treinamento pré-temporada de jogadores de elite da liga de rugby australiano. Diante do exposto e a ausência de estudos padronizados que correlacionam a ação do TT no ganho de peso, o Instituto Australiano do Esporte não recomenda sua utilização devido à escassez de evidências que comprovam sua segurança e eficácia (Pokrywka et al., 2014).

O uso de plantas medicinas na prevenção de hipertrofia cardíaca (HC) é difundido na literatura. Sankar et al. (2013) expõe em seu experimento, efeitos positivos no controle do remodelamento cardíaco com uso de Desmodium gangeticum tradicionalmente na Índia. Prathapan et al. (2014) demonstram efetividade da Boerhaavia diffusa ('Erva-tostão'), contra a disfunção mitocondrial na HC induzida pela angiotensina II em cardiomioblastos H9c2. Por sua vez, Guo et al. (2007) e Reshma et al. (2016) demostraram que o TT melhorou o perfil lipídico em ratos e também preveniu o remodelamento do VE e melhorou a função cardíaca e disfunção mitocondrial na fase inicial pós infarto de ratos hiperlipidêmicos. A ação do TT na prevenção de eventos cardiovasculares isquêmicos em modelos in vitro (linha celular H9c2) e in vivo (rato Wistar) atuando na via antiapoptótica por meio do seu potencial antioxidante (Reshma et al. 2015; Reshma et al. 2019). No presente estudo em ratos íntegros, sem alterações no perfil lipídico, observamos que o TT na dose de 50mg/kg/dia não afetou a proporção PC/PA, no diâmetro dos cardiomiócitos e depósito de colágeno ventricular. Sendo assim, qualquer alteração na histomorfologia no coração e na aorta causada pelo uso da TT é um efeito considerável e que deve ser melhor compreendido, visto que, alterações estruturais nestes órgãos podem impedir seu adequado funcionamento e afetar gravemente a eficiência do bombeamento cardíaco, podendo levar até mesmo o óbito.

Atualmente, o TT é amplamente utilizado por atletas e fisiculturistas que visam na melhoria do desempenho, força muscular e aumento nas concentrações de testosterona. No entanto, por mais que o existem várias declarações de marketing em torno da planta afrodisíaca, o uso da TT e os seus efeitos no organismo ainda é um assunto muito debatido e controverso, principalmente no que diz respeito a um efeito androgênico na força muscular e composição corporal (Lemos Júnior et al., 2011; Neychev et al., 2016). Alguns fabricantes referem que o uso da erva não levará a um teste hormonal positivo em atletas, outros dizem que seu uso pode aumentar a taxa de testosterona/epitestosterona urinária (Lemos Júnior et al., 2011). No 
presente estudo observou que o TT não afeta a secreção de testosterona e a variação do peso corporal apresentada nos ratos tratados com TT não podem ser relacionar com efeito androgênico. Esses resultados corrobolam com os estudos de Rogerson et al. (2007) que demonstraram que a suplementação com TT não contribuiu para o aumento da massa muscular e da produção de testosterona em atletas. Além disso, segundo uma revisão sistemática sobre o extrato de ervas TT proposta por Qureshi et al. (2014), a associação da planta com o aumento das concentrações de testosterona é equivocada. Salienta-se, ainda, que o marketing em torno dos seus efeitos nos níveis de testosterona em humanos não possuem evidências plausíveis e seriam suplantadas pela liberação de óxido nítrico, independente dos níveis de testosterona sérica. Estudos demonstraram que pode haver um benefício do uso do TT no desempenho aeróbico de atletas e ratos com um mecanismo adjacente associado ao fator de crescimento da insulina, mas não ao efeito andrógeno no plasma (Yin et al., 2016; Ma et al., 2017; Wu et al., 2017).

Apesar da ausência de evidências da relação do TT com os níveis plasmáticos de testosterona, estudos sugerem efeitos vantajosos sobre os parâmetros do esperma (Santos et al., 2019). Pesquisas etnobotânicas veinculam o uso de TT no tratamento de disfunção erétil masculina e feminina, com resultados conflitantes (Malviya et al., 2011; Malviya et al., 2016; Prescott et al., 2020). O aumento da motilidade dos espermatozoides neste estudo foi determinado pela análise comparativa do espermograma e corrobora com os estudos de Khaleghi et al. (2017) no qual o uso de extrato de TT, in vitro, aumentou significativamente a motilidade total do esperma. O estudo de Salgado et al. (2017) com sessenta e cinco homens inférteis em uso de Androsten (® também demonstraram efeitos positivos em várias parâmetros, incluindo motilidade. No entanto, possui evidências conflitantes com o estudo de Roaiah et al. (2017) em que não foram observadas diferenças significativas no parâmetro do sêmen em homens com infertilidade idiopática. Diante disso, tais resultados devem ser avaliados com cautela. Em uma revisão sistemática proposta por Sanagoo et al. (2019), com a mesma temática do estudo de Roaiah et al. (2017), 6 dos 7 artigos incluídos apontavam, de maneira geral, melhora dos parâmetros espermáticos. Diante disso, o TT demonstra ser uma terapêutica alternativa promissora em casos de infertilidade masculina, principalmente em disfunções de motilidade (Asadmobini et al., 2017). Contudo, alguns estudos limitados destacam o risco de nefrotoxicidade após a administração de suplementos de TT (Ștefănescu et al., 2020). No entanto, mais estudos são necessários para esclarecer tais aspectos toxicológicos ou comprovar sua segurança.

\section{Conclusão}

Nesse protocolo experimental, com a dose de $50 \mathrm{mg} / \mathrm{kg}$ em ratos, o TT pode ter um futuro promissor em preservar o peso corporal, sem alterar a histomorfologia cardíaca e fisiologia testicular de ratos. Contudo, salienta-se que há uma escassez de estudos que avaliem os efeitos terapêuticos do TT em humanos. Por isso, é importante investir em pesquisas, principalmente ensaios clínicos, que esclareçam o modo de preparação, administração, efeitos farmacológico e, principalmente, sua segurança e eficácia.

\section{Referências}

Antonio, J., Uelmen, J., Rodriguez, R., \& Earnest, C. (2000). The effects of Tribulus terrestris on body composition and exercise performance in resistancetrained males. International journal of sport nutrition and exercise metabolism, 10(2), 208-215. https://doi.org/10.1123/ijsnem.10.2.208.

Arcasoy, H. B., Erenmemisoglu, A., Tekol, Y., Kurucu, S., \& Kartal, M. (1998). Effect of Tribulus terrestris L. saponin mixture on some smooth muscle preparations: a preliminary study. Bollettino chimico farmaceutico, 137(11), 473-475.

Armstrong, L. E., Soto, J. A., Hacker, F. T., Jr, Casa, D. J., Kavouras, S. A., \& Maresh, C. M. (1998). Urinary indices during dehydration, exercise, and rehydration. International journal of sport nutrition, 8(4), 345-355. https://doi.org/10.1123/ijsn.8.4.345.

Asadmobini, A., Bakhtiari, M., Khaleghi, S., Esmaeili, F., \& Mostafaei, A. (2017). The effect of Tribulus terrestris extract on motility and viability of human sperms after cryopreservation. Cryobiology, 75, 154-159. https://doi.org/10.1016/j.cryobiol.2017.02.005. 
Brown, G. A., Vukovich, M. D., Reifenrath, T. A., Uhl, N. L., Parsons, K. A., Sharp, R. L., \& King, D. S. (2000). Effects of anabolic precursors on serum testosterone concentrations and adaptations to resistance training in young men. International journal of sport nutrition and exercise metabolism, 10(3), 340 359. https://doi.org/10.1123/ijsnem.10.3.340.

Chhatre, S., Nesari, T., Somani, G., Kanchan, D., \& Sathaye, S. (2014). Phytopharmacological overview of Tribulus terrestris. Pharmacognosy reviews, 8(15), 45-51. https://doi.org/10.4103/0973-7847.125530.

Do, J., Choi, S., Choi, J., \& Hyun, J. S. (2013). Effects and Mechanism of Action of a Tribulus terrestris Extract on Penile Erection. Korean journal of urology, 54(3), 183-188. https://doi.org/10.4111/kju.2013.54.3.183.

GamalEl Din, S. F., Abdel Salam, M. A., Mohamed, M. S., Ahmed, A. R., Motawaa, A. T., Saadeldin, O. A., \& Elnabarway, R. R. (2019). Tribulus terrestris versus placebo in the treatment of erectile dysfunction and lower urinary tract symptoms in patients with late-onset hypogonadism: A placebo-controlled study. Urologia, 86(2), 74-78. https://doi.org/10.1177/0391560318802160.

Gauthaman, K., \& Ganesan, A. P. (2008). The hormonal effects of Tribulus terrestris and its role in the management of male erectile dysfunction--an evaluation using primates, rabbit and rat. Phytomedicine: international journal of phytotherapy and phytopharmacology, 15(1-2), 44-54. https://doi.org/10.1016/j.phymed.2007.11.011.

Gauthaman, K., Adaikan, P. G., \& Prasad, R. N. (2002). Aphrodisiac properties of Tribulus Terrestris extract (Protodioscin) in normal and castrated rats. Life sciences, 71(12), 1385-1396. https://doi.org/10.1016/s0024-3205(02)01858-1.

Guo, Y., Shi, D. Z., Yin, H. J., \& Chen, K. J. (2007). Effects of Tribuli saponins on ventricular remodeling after myocardial infarction in hyperlipidemic rats. The American journal of Chinese medicine, 35(2), 309-316. https://doi.org/10.1142/S0192415X07004837.

Hussain A., Abbas A. Mohammed, Heba. H. Ibrahim, \& Amir H. Abbas. (2009). Study the Biological Activities of Tribulus Terrestris Extracts. 3:9-24. http://doi.org/10.5281/zenodo.1085151.

Junqueira, L. C., Bignolas, G., \& Brentani, R. R. (1979). Picrosirius staining plus polarization microscopy, a specific method for collagen detection in tissue sections. The Histochemical journal, 11(4), 447-455. https://doi.org/10.1007/BF01002772.

Khaleghi, S., Bakhtiari, M., Asadmobini, A., \& Esmaeili, F. (2017). Tribulus terrestris Extract Improves Human Sperm Parameters In Vitro. Journal of evidence-based complementary \& alternative medicine, 22(3), 407-412. https://doi.org/10.1177/2156587216668110.

Kohut, M. L., Thompson J. R., Campbell J., Brown G. A., Vukovich M. D., Jackson D. A., \& King D. S. (2003). Ingestion of a dietary supplement containing dehydroepiandrosterone (DHEA) and androstenedione has minimal effect on immune function in middle-aged men. Journal of the American College of Nutrition. 22:363-371. https://doi.org/10.1080/07315724.2003.10719319.

Lemos Júnior L. P. H., Lemos A. L. A., Lemos L. M. D. (2011). Tribulus terrestris. Diagn Tratamento. 16:170-173.

Ma, Y., Guo, Z., \& Wang, X. (2017). Tribulus terrestris extracts alleviate muscle damage and promote anaerobic performance of trained male boxers and its mechanisms: Roles of androgen, IGF-1, and IGF binding protein-3. Journal of sport and health science, 6(4), 474-481. https://doi.org/10.1016/j.jshs.2015.12.003.

Malviya, N., Jain, S., Gupta, V. B., \& Vyas, S. (2011). Recent studies on aphrodisiac herbs for the management of male sexual dysfunction---a review. Acta poloniae pharmaceutica, 68(1), 3-8.

Malviya, N., Malviya, S., Jain, S., \& Vyas, S. (2016). A review of the potential of medicinal plants in the management and treatment of male sexual dysfunction. Andrologia, 48(8), 880-893. https://doi.org/10.1111/and.12677.

Neychev, V., \& Mitev, V. (2016). Pro-sexual and androgen enhancing effects of Tribulus terrestris L.: Fact or Fiction. Journal of ethnopharmacology, 179, 345-355. https://doi.org/10.1016/j.jep.2015.12.055.

Pereira, A. S., Shitsuka, D. M., Parreira, F. J., \& Shitsuka, R. (2018). Metodologia da pesquisa científica. UFSM. https://repositorio.ufsm.br/bitstream/ handle/1/15824/Lic_Computacao_Metodologia-Pesquisa-Cientifica.pdf?sequence=1.

Pokrywka, A., Obmiński, Z., Malczewska-Lenczowska, J., Fijałek, Z., Turek-Lepa, E., \& Grucza, R. (2014). Insights into Supplements with Tribulus Terrestris used by Athletes. Journal of human kinetics, 41, 99-105. https://doi.org/10.2478/hukin-2014-0037.

Prathapan, A., Vineetha, V. P., \& Raghu, K. G. (2014). Protective effect of Boerhaavia diffusa L. against mitochondrial dysfunction in angiotensin II induced hypertrophy in H9c2 cardiomyoblast cells. PloS one, 9(4), e96220. https://doi.org/10.1371/journal.pone.0096220.

Prescott, H., \& Khan, I. (2020). Medicinal plants/herbal supplements as female aphrodisiacs: Does any evidence exist to support their inclusion or potential in the treatment of FSD? Journal of ethnopharmacology, 251, 112464. https://doi.org/10.1016/j.jep.2019.112464.

Qureshi, A., Naughton, D. P., \& Petroczi, A. (2014). A systematic review on the herbal extract Tribulus terrestris and the roots of its putative aphrodisiac and performance enhancing effect. Journal of dietary supplements, 11(1), 64-79. https://doi.org/10.3109/19390211.2014.887602.

Reshma, P. L., Binu, P., Anupama, N., Vineetha, R. C., Abhilash, S., Nair, R. H., \& Raghu, K. G. (2019). Pretreatment of Tribulus terrestris L. causes antiischemic cardioprotection through MAPK mediated anti-apoptotic pathway in rat. Biomedicine \& Pharmacotherapy = Biomedecine \& Pharmacotherapie, 111, 1342-1352. https://doi.org/10.1016/j.biopha.2019.01.033.

Reshma, P. L., Lekshmi, V. S., Sankar, V., \& Raghu, K. G. (2015). Tribulus terrestris (Linn.) Attenuates Cellular Alterations Induced by Ischemia in H9c2 Cells Via Antioxidant Potential. Phytotherapy research: PTR, 29(6), 933-943. https://doi.org/10.1002/ptr.5336.

Reshma, P. L., Sainu, N. S., Mathew, A. K., \& Raghu, K. G. (2016). Mitochondrial dysfunction in H9c2 cells during ischemia and amelioration with Tribulus terrestris L. Life sciences, 152, 220-230. https://doi.org/10.1016/j.lfs.2016.03.055. 
Roaiah, M. F., Elkhayat, Y. I., Abd El Salam, M. A., \& Din, S. (2017). Prospective Analysis on the Effect of Botanical Medicine (Tribulus terrestris) on Serum Testosterone Level and Semen Parameters in Males with Unexplained Infertility. Journal of dietary supplements, 14(1), 25-31. https://doi.org/10.1080/19390211.2016.1188193.

Rogerson, S., Riches, C. J., Jennings, C., Weatherby, R. P., Meir, R. A., \& Marshall-Gradisnik, S. M. (2007). The effect of five weeks of Tribulus terrestris supplementation on muscle strength and body composition during preseason training in elite rugby league players. Journal of strength and conditioning research, 21(2), 348-353. https://doi.org/10.1519/R-18395.1.

Salgado, R. M., Marques-Silva, M. H., Gonçalves, E., Mathias, A. C., Aguiar, J. G., \& Wolff, P. (2017). Effect of oral administration of Tribulus terrestris extract on semen quality and body fat index of infertile men. Andrologia, 49(5), 10.1111/and.12655. https://doi.org/10.1111/and.12655.

Samaha, A. A., Nasser-Eddine, W., Shatila, E., Haddad, J. J., Wazne, J., \& Eid, A. H. (2008). Multi-organ damage induced by anabolic steroid supplements: a case report and literature review. Journal of medical case reports, 2, 340. https://doi.org/10.1186/1752-1947-2-340.

Samani, N. B., Jokar, A., Soveid, M., Heydari, M., \& Mosavat, S. H. (2016). Efficacy of the Hydroalcoholic Extract of Tribulus terrestris on the Serum Glucose and Lipid Profile of Women With Diabetes Mellitus: A Double-Blind Randomized Placebo-Controlled Clinical Trial. Journal of evidence-based complementary \& alternative medicine, 21(4), NP91-NP97. https://doi.org/10.1177/2156587216650775.

Sanagoo, S., Sadeghzadeh Oskouei, B., Gassab Abdollahi, N., Salehi-Pourmehr, H., Hazhir, N., \& Farshbaf-Khalili, A. (2019). Effect of Tribulus terrestris L. on sperm parameters in men with idiopathic infertility: A systematic review. Complementary therapies in medicine, 42, 95-103. https://doi.org/10.1016/j.ctim.2018.09.015.

Sankar, V., Pangayarselvi, B., Prathapan, A., \& Raghu, K. G. (2013). Desmodium gangeticum (Linn.) DC. exhibits antihypertrophic effect in isoproterenolinduced cardiomyoblasts via amelioration of oxidative stress and mitochondrial alterations. Journal of cardiovascular pharmacology, 61(1), 23-34. https://doi.org/10.1097/FJC.0b013e3182756ad3

Santos, H. O., Howell, S., \& Teixeira, F. J. (2019). Beyond tribulus (Tribulus terrestris L.): The effects of phytotherapics on testosterone, sperm and prostate parameters. Journal of Ethnopharmacology, 235, 392-405. https://doi.org/10.1016/j.jep.2019.02.033.

Shahid, M., Riaz, M., Talpur, M. M., \& Pirzada, T. (2016). Phytopharmacology of Tribulus terrestris. Journal of biological regulators and homeostatic agents, 30(3), 785-788.

Shirani, J., Pick, R., Roberts, W. C., \& Maron, B. J. (2000). Morphology and significance of the left ventricular collagen network in young patients with hypertrophic cardiomyopathy and sudden cardiac death. Journal of the American College of Cardiology, 35(1), 36-44. https://doi.org/10.1016/s07351097(99)00492-1.

Souza, A. da S., Ribeiro Junior, O. C., Ribeiro, J. S. S., Mendonça, L. B., Melo, J. M. R. de, \& Araújo, T. S. de. (2020). The use of phytotherapy in the management of women in climacteric/menopause. Research, Society and Development, 9(9), e415997416. https://doi.org/10.33448/rsd-v9i9.7416.

Ștefănescu, R., Tero-Vescan, A., Negroiu, A., Aurică, E., \& Vari, C. E. (2020). A Comprehensive Review of the Phytochemical, Pharmacological, and Toxicological Properties of Tribulus terrestris L. Biomolecules, 10(5), 752. https://doi.org/10.3390/biom10050752.

Wang, B., Ma, L., \& Liu, T. (1990). Zhong xi yi jie he za zhi = Chinese journal of modern developments in traditional medicine, 10(2), 85-68.

Wu, Y., Yang, H., \& Wang, X. (2017). The function of androgen/androgen receptor and insulin growth factor-1/insulin growth factor-1 receptor on the effects of Tribulus terrestris extracts in rats undergoing high intensity exercise. Molecular medicine reports, 16(3), 2931-2938. https://doi.org/10.3892/mmr.2017.6891.

Yin, L., Wang, Q., Wang, X., \& Song, L. N. (2016). Effects of Tribulus terrestris saponins on exercise performance in overtraining rats and the underlying mechanisms. Canadian journal of physiology and pharmacology, 94(11), 1193-1201. https://doi.org/10.1139/cjpp-2016-0086.

Yuan, Z., Du, W., He, X., Zhang, D., \& He, W. (2020). Tribulus terrestris Ameliorates Oxidative Stress-Induced ARPE-19 Cell Injury through the PI3K/AktNrf2 Signaling Pathway. Oxidative medicine and cellular longevity, 2020, 7962393. https://doi.org/10.1155/2020/7962393.

Zhang, H., Tong, W. T., Zhang, C. R., Li, J. L., Meng, H., Yang, H. G., \& Chen, M. (2019). Gross saponin of Tribulus terrestris improves erectile dysfunction in type 2 diabetic rats by repairing the endothelial function of the penile corpus cavernosum. Diabetes, metabolic syndrome and obesity: targets and therapy, 12, 1705-1716. https://doi.org/10.2147/DMSO.S205722. 